\title{
Medical Or Surgical Treatment In Cryptographic Stroke With Pattent Foramen Oval (PFO)
}

Athena Sharifi Razavi,.

Assistant Professor in Neurology, M azandaran University of M edical Sciennce, Sari, Iran.

PFO is one of common congenital cardiac anomalies. It's incidence is $15-30 \%$ and decreases with age.

PFO is associated with several disease processes such as transient ischemic attacks and stroke. Various studies have reported that $45-54 \%$ of patients with cryptogenic stroke have PFO.

The underlying pathological mechanism of PFO-associated stroke is the passage of abnormal material from the venous circulation through the PFO into the systemic circulation.

As individuals age and develop comorbid cardiovascular disease such as heart failure or diastolic dysfunction the propensity to shunt right to left may increase and the risk of hypercoaguability may increase as well. Thus PFO only become pathogenic in the setting of certain comorbid conditions that cause the formation of phatogenic material with embolic potential.

Controversy exists regarding closure of PFO as a therapeutic treatment modality for cryptogenic stroke.

In properly selected patient closure of PFO is a safe and effective treatment modality. Contemporary indication for PFO closure in stroke or TIA is: 1.Documented paradoxical embolus. 2. Recurrent cryptogenic events. 3. Single cryptogenic event and inability to tolerate medical therapy. 4. Single cryptogenic event with high risk anatomical PFO features.

Key words: PFO, stroke

DOI: $10.7575 /$ aiac.abcmed.ca1.43

Published Date: February 2017

Peer-review is under responsibility of the 9th Iranian Stroke Congress.

Published by Australian International Academic Centre, Australia

This published work is open access under the CC BY license.

Available online at www.abcmed.aiac.org.au 